\title{
Aortic distensibility and stiffness index measured by magnetic resonance imaging in patients with Marfan's syndrome
}

\author{
J N Adams, M Brooks, T W Redpath, F W Smith, J Dean, J Gray, S Walton, R J Trent
}

Cardiac Department, Aberdeen Royal Infirmary

J N Adams

$\mathrm{S}$ Walton

R J Trent

Department of

Radiology, Aberdeen

Royal Infirmary

M Brooks

Magnetic Resonance

Imaging Centre,

Aberdeen Royal

Infirmary

T W Redpath

F W Smith

Department of

Genetics, Aberdeen

University

J Dean

Molecular Genetics,

Department of

Pathology, Ninewells

Hospital, Dundee

J Gray

Correspondence to:

Dr J N Adams, The Ay

Hospital, Dalmellingto

Road, Ayr KA6 6XD.

Accepted for publication

29 September 1994

\begin{abstract}
Objectives-To use magnetic resonance imaging to measure the elastic properties of the aorta of adults with Marfan's syndrome and to compare these results with those obtained by echocardiography. Patients and methods -12 patients with Marfan's syndrome and 12 controls matched for age. Transverse luminal areas of the ascending and descending aorta were measured using electrocardiographic gated magnetic resonance imaging. Echocardiography was used to measure the diameter of the ascending aorta and aortic arch in patients with Marfan's syndrome. Blood pressure was measured during both scans.

Results-In diastole, transverse luminal areas of the ascending and descending aorta were significantly greater in patients with Marfan's syndrome when measured by magnetic resonance imaging and corrected for body surface area; $P<0.02$ and $P<0.05$ respectively. Patients with Marfan's syndrome had a higher stiffness index $(112 \cdot 77 v 5 \cdot 78, P<$ $0.05)$ and a lower distensibility (0.0066 $v$ $0.0105, P<0.05)$ than controls. Results produced by MRI and echocardiography were not significantly different.

Conclusions-Magnetic resonance imaging gives good quality reproducible images of the ascending and descending aorta. In patients with Marfan's syndrome, aortic distensibility and stiffness index measured by magnetic resonance imaging were abnormal (but did not always relate directly to the size of the aorta.
\end{abstract}

(Br Heart f 1995;73:265-269)

Keywords: Marfan's syndrome; magnetic resonance imaging; aortic elastic properties

Marfan's syndrome is an inherited connective tissue disorder chiefly affecting the eyes, skeleton, and cardiovascular system. ${ }^{1-3}$ Recent genetic and immunohistochemical findings suggest that the defect is in fibrillin, a microfibrillar protein abundant in the tissues affected in Marfan's syndrome. ${ }^{4}$ The cardiovascular abnormalities include mitral valve prolapse; mitral regurgitation; aortic regurgitation; and aortic dilatation, dissection, and rupture. $^{1-3}$ Complications involving the thoracic aorta are the main cause of death in patients with Marfan's syndrome. ${ }^{5}$ Angiography, ${ }^{6}$ echocardiography, ${ }^{23}$ and magnetic resonance imaging, ${ }^{7}$ have all been used to measure aortic size in patients with Marfan's syndrome.

Non-invasive techniques can measure the distensibility and stiffness of the aortic wall ${ }^{8}$; echocardiography has been used to measure the abnormal elastic properties of the aorta in patients with Marfan's syndrome ${ }^{10}$ and magnetic resonance imaging has been used to measure aortic distensibility in children with Marfan's syndrome. ${ }^{11}$ The aim of this study was to use magnetic resonance imaging to measure aortic distensibility and stiffness index in adults with Marfan's syndrome and compare the results with those obtained by echocardiography.

\section{Patients and methods}

We studied 12 patients (eight men and four women) (aged 19-56, mean 34.1) who fulfilled the strict diagnostic criteria for Marfan's syndrome. ${ }^{12}$ All medication which could interfere with measurements was stopped for at least five half lives and patients with a history of aortic dissection or surgery were excluded. Twelve age matched subjects (range 19 to 56 years; mean 36.5 years), six men and six women, with no history of heart disease formed the control group. There was no significant difference in age or body surface area between the two groups. The protocol had been approved by the local ethics committee.

We used a Siemens 0.95T Impact magnetic resonance scanner to obtain oblique transverse images transecting the aortic arch as described by Mohiaddin et al. ${ }^{13} \mathrm{We}$ identified the ascending and descending aorta by obtaining a multislice transverse image set, and then selected the image best depicting the complete aortic arch using a multislice oblique sagittal set. Thus a single slice spin echo $(\mathrm{TE}=25 \mathrm{~ms}$ ) image could be positioned perpendicular to the ascending and descending aorta at the level of the pulmonary artery. Images were electrocardiogram gated such that end diastolic images were acquired 100 $\mathrm{ms}$ before the average RR interval and end systolic images at the end of the $T$ wave. An Odam Bruker Maglife automatic monitor measured blood pressure at the brachial artery while the patient was in the scanner. Systolic and diastolic blood pressure were measured directly before their respective images were acquired. The aortic lumen was outlined manually on the computer screen on two 

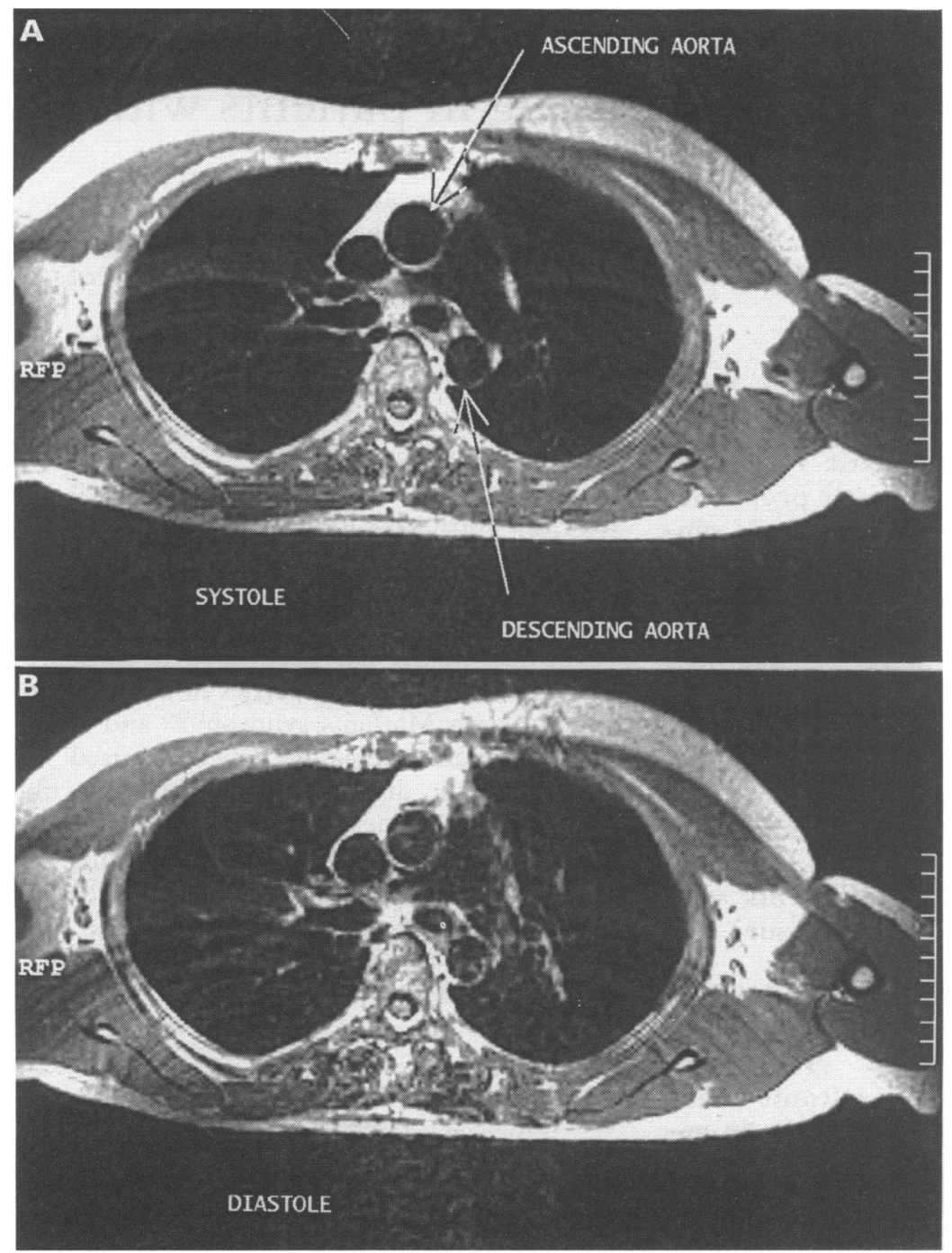

Figure 1 MRI images of ascending and descending aorta at the bifurcation of the pulmonary artery in a 32 year old male control in $(A)$ systole and $(B)$ diastole. RFP, right foot posterior; field of view $450 \times 450 \mathrm{~mm}$.

occasions by two operators blinded to each others results.

Echocardiograms were performed on the same day with a Vingmed (CFM 700E) with patients in the left lateral position. We measured the systolic and diastolic diameters of the ascending aorta $3 \mathrm{~cm}$ above the aortic valve using two dimensional $M$ mode tracings from long axis parasternal views. Similarly the aortic arch was measured at a level just before the origin of the left common carotid artery from suprasternal views with the patient

Mean aortic area, distensibility and stiffness index measured by MRI

\begin{tabular}{lcll}
\hline Variable & Patients $(n=12)$ & Controls $(n=12)$ & $P^{*}$ \\
\hline Mean body surface area $\left(\mathrm{m}^{2}\right)$ & 1.99 & 1.85 & $\mathrm{NS} \dagger$ \\
Ascending aorta: & 9.20 & 7.31 & $<0.01$ \\
Systolic area $\left(\mathrm{cm}^{2}\right)$ & 7.94 & 5.89 & $<0.01$ \\
Diastolic area $\left(\mathrm{cm}^{2}\right)$ & 3.96 & 3.20 & $<0.02$ \\
Corrected area $\left(\mathrm{cm}^{2} / \mathrm{m}^{2}\right) \ddagger$ & 0.0066 & 0.0105 & $<0.05$ \\
Distensibility $\left(\mathrm{mm} \mathrm{Hg}^{-1}\right)$ & 112.77 & 5.78 & $<0.05$ \\
Stiffness index & 5.99 & 4.52 & $<0.01$ \\
Descending aorta: & 5.30 & 3.73 & $<0.01$ \\
Systolic area $\left(\mathrm{cm}^{2}\right)$ & 2.65 & 2.03 & $<0.05$ \\
Diastolic area $\left(\mathrm{cm}^{2}\right)$ & 0.0060 & 0.0120 & $<0.05$ \\
Corrected area $\left(\mathrm{cm}^{2} / \mathrm{m}^{2}\right) \ddagger$ & 50.23 & 5.77 & $<0.05$ \\
Distensibility $\left(\mathrm{mm} \mathrm{Hg}^{-1}\right)$ & Stiffness index & &
\end{tabular}

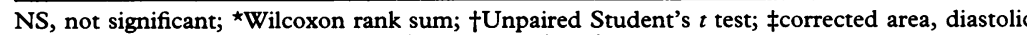
area/body surface area; MRI, magnetic resonance imaging. supine. The average of three measurements was taken. A Bard automatic blood pressure monitor measured blood pressure at the brachial artery immediately before and after the measurements were made. Pulse pressure was calculated as the difference between systolic and diastolic blood pressure.

Aortic distensibility was calculated by the following formula:

distensibility $=2$ (aortic luminal area change) $/$ (diastolic luminal area $\times$ pulse pressure) ${ }^{8}$ For echocardiographic measurements we used the aortic diameter rather than the aortic area. The pressure-independent aortic stiffness index was calculated using:

stiffness = In (systolic/diastolic blood pressure)/(aortic diameter change/aortic diastolic diameter). ${ }^{9}$ Diameters derived from the magnetic resonance imaging area measurements assumed that the aorta is circular.

\section{STATISTICS}

Intra observer and inter observer reproducibility were calculated as the standard deviation of the differences between measurements expressed as percentage of the mean of the measurements. An unpaired Student's $t$ test was used for normal distributions and non parametric testing was performed using a Wilcoxon rank sum test.

\section{Results}

MAGNETIC RESONANCE IMAGING

Figure 1 shows systolic and diastolic images for a 32 year old male control. Patients with Marfan's syndrome had significantly greater ascending and descending aortic areas than the control group $(P<0.01)$. When diastolic areas were corrected for body surface area this difference persisted $(P<0.02$ ascending aorta and $P<0.05$ descending aorta) (table). The aortic stiffness index in the ascending and descending aorta was significantly greater in patients with Marfan's syndrome (table). At both sites distensibility was reduced and stiffness index increased with increasing age of controls and patients with Marfan's syndrome. With increasing aortic area there was a trend towards low distensibility and high stiffness indices. This was not, however, the case for all patients (fig 2).

Intra observer reproducibility ranged from $2.1 \%$ for the ascending aorta in diastole to $3.8 \%$ for the descending aorta in diastole. Similarly interobserver reproducibility ranged from $4.0 \%$ in the ascending aorta in systole to $5 \cdot 2 \%$ in the descending aorta in systole. Reproducibility was lower for the descending aorta and diastolic images. The mean percentage change of aortic area was $16 \%$ in the ascending aorta and $8 \%$ in the descending aorta. Intra and inter observer reproducibilities were within acceptable limits.

\section{COMPARISON WITH ECHOCARDIOGRAPHY}

Figure 3 shows the relation between systolic aortic diameter measured by echocardiography and distensibility and stiffness index for patients with Marfan's syndrome. To enable 
Figure 2 (A) Relation between aortic area and aortic distensibility and (B) relation between aortic area and aortic stiffness measured by MRI in patients with Marfan's syndrome. Data on one patient whose stiffness index was too high for the scale are not shown.

Figure 3 (A) Relation between aortic diameter and aortic distensibility and $(B)$ relation between aortic area and stiffness index measured by echocardiography in patients with Marfan's syndrome. Data on one patient whose stiffness index was too high for the scale are not shown.
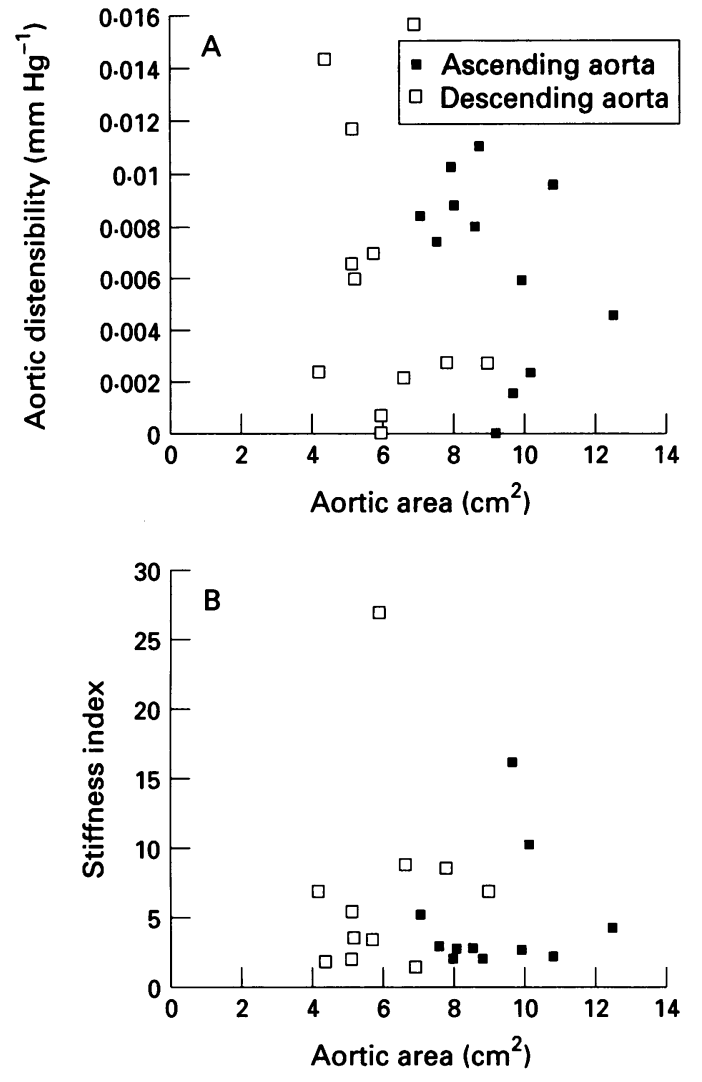

direct comparison with the echocardiographic measurements aortic diameters were derived from the magnetic resonance imaging area measurements. There was no significant difference in the aortic diameters, distensibility, or stiffness indices measured by the two tech-
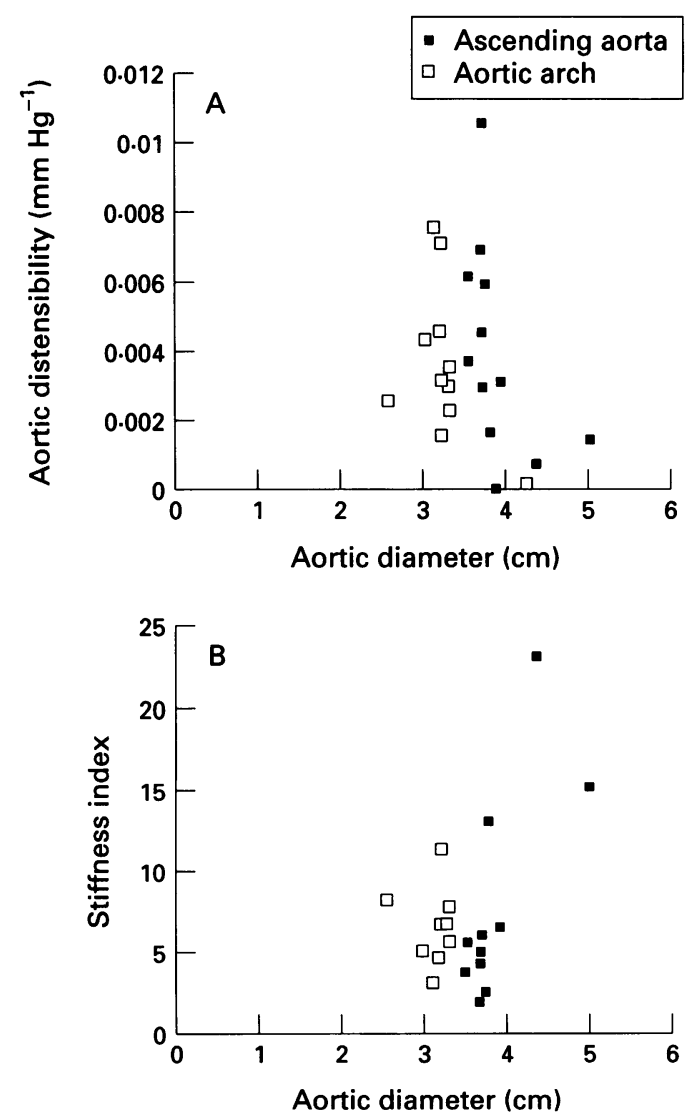

niques. Figure 4 compares aortic distensibility and stiffness index measured by the two techniques for individual patients.

\section{PATIENT ACCEPTABILITY}

Fourteen patients with Marfan's syndrome were identified as suitable for this study. Two declined to take part because of claustrophobia. Of the 12 patients in the study five expressed a preference for the magnetic resonance imaging scan, five for the echocardiogram, and two had no preference.

\section{Discussion}

In this series magnetic resonance imaging measurements of patients with Marfan's syndrome showed larger luminal areas of the aorta, lower aortic distensibilities, and higher stiffness indices than controls of a similar age. These findings using magnetic resonance imaging are similar to those of Savolainen et $a l^{11}$ in children with Marfan's syndrome and Hirata et $a l^{10}$ using echocardiography. Direct comparison with these studies is difficult, however, because of differences in the site at which measurements were taken along the aorta and the age of patients studied. Other workers reported that aortic area and stiffness index increase and distensibility decreases with increasing age and coronary artery disease. ${ }^{7813}$ In this study the controls were matched for age and no subject had a history of coronary artery disease.

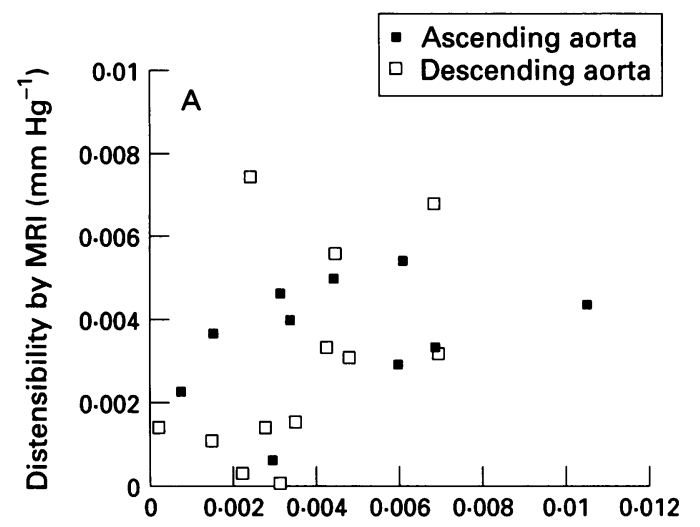

Distensibility by echocardiogram $\left(\mathrm{mm} \mathrm{Hg}^{-1}\right.$ )

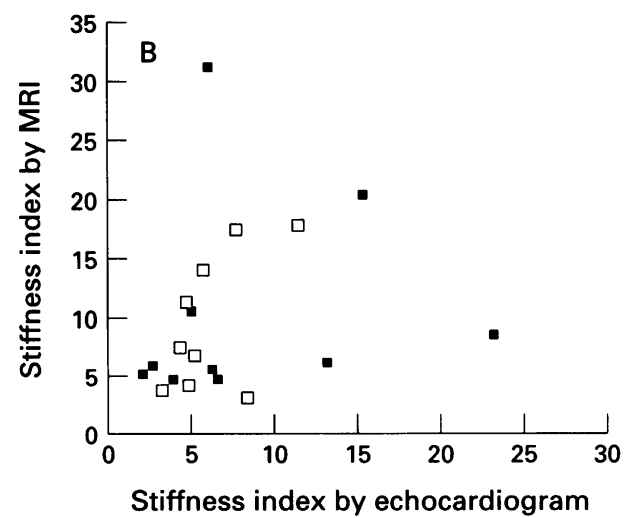

Figure 4 Comparisons of $(A)$ aortic distensibility measured by echocardiography and $M R I$ and $(B)$ aortic stiffness index measured by echocardiography and MRI in patients with Marfan's syndrome. Data on two patients whose stiffness indices were too high for the scale are not shown. 
To compare distensibility measured by magnetic resonance imaging and echocardiography it is necessary to convert area measurements to diameters or vice versa. Because the conversion assumes that the aorta is circular it is a possible source of error. Although there was no overall significant difference in aortic distensibility or stiffness index when measured by echocardiogram or magnetic resonance imaging, there was a large difference in some individual patients in some cases (fig 4). In two cases this may be because it was difficult to obtain satisfactory echocardiogram images. Anatomical and technical factors prevent imaging of the descending thoracic aorta by echocardiography, but it is possible to obtain echocardiographic images of the aortic arch and the abdominal aorta. Although it is possible to measure the area of the aortic arch by magnetic resonance imaging this would require further images thereby increasing the time in the scanner. In this study we compared echocardiographic measurements from the aortic arch with magnetic resonance imaging measurements from the descending thoracic aorta. There was no statistical difference in these two measurements in patients with Marfan's syndrome.

Although the relation between fibrillin gene abnormalities and Marfan's syndrome is becoming clearer, studies of phenotypegenotype correlations are at an early stage. ${ }^{14}$ Knowledge of the gene mutation, details of the cellular synthesis and of the secretion or incorporation of fibrillin into microfibrils can not yet be used to predict the risk of cardiovascular complications. As medical ${ }^{15}$ and surgical $^{1617}$ intervention in Marfan's syndrome, can be beneficial, it is necessary to look for other predictors of severity to direct appropriate treatment.

The current recommendation is that patients with Marfan's syndrome should have their aortic size monitored so that prophylactic surgery can be considered when the aortic diameter exceeds $55 \mathrm{~mm}^{18}{ }^{19}$ (this is equivalent to an area of $23.8 \mathrm{~cm}^{2}$ if the aorta is circular). Aortic dissection can, however, occur in Marfan's syndrome before this limit is reached or even in the absence of aortic dilatation. ${ }^{1}$ In this series no patients had an aortic systolic diameter $>52 \mathrm{~mm}$ measured by echocardiogram or a systolic area $>12.5 \mathrm{~cm}^{2}$ by magnetic resonance imaging. Figures 2 and 3 show that some patients with relatively small aortic diameters or areas have very low distensibilities and high stiffness indices in the ascending aorta. The abnormal microfibrillar fibres present in Marfan's syndrome are found in the aortic tunica media ${ }^{4}$ and histopathological examination shows disruption of collagen fibres and cystic medial necrosis. ${ }^{20}{ }^{21}$ Schlatmann et $a l^{20}$ showed that in patients with Marfan's syndrome the intrinsically weakened wall of the aorta is easily injured and therefore predisposed to dissection. Thus measurements of aortic distensibility and stiffness index may be useful to detect aortic involvement before the aorta becomes significantly dilated. At present, however, no data are available to assess the usefulness of aortic distensibility or stiffness index as a predictor of aortic rupture or dissection in patients with Marfan's syndrome. Further work is needed to establish the place of such measurements in the management of Marfan's syndrome.

If serial measurements of aortic size or elastic properties are to be made it is important to use the same aortic site on each occasion. The use of the bifurcation of the pulmonary artery to align magnetic resonance imaging images ensures that measurements can be made at the same level on each occasion. Careful positioning of magnetic resonance imaging images also ensures that measurements are made perpendicular to the lumen of the aorta. Anatomical landmarks can also be used to ensure that echocardiographical measurements are repeated at the same site, although this can be difficult owing to the quality of echocardiogram images and the often limited field of view. In this study most technical difficulties were experienced in obtaining echocardiogram images, in particular images $3 \mathrm{~cm}$ above the aortic valve. Chest wall deformities are common in Marfan's syndrome and this may compound the problems of obtaining accurate echocardiogram measurements. In Marfan's syndrome the ascending aorta is normally the first area to become dilated, but the aortic arch and descending aorta may also be affected. As a preliminary to obtaining the magnetic resonance imaging images of the aorta, from which area measurements are made, it is necessary to acquire a multislice oblique sagittal set. At least one of these images normally depicts the complete thoracic aorta and thus provides an opportunity to assess the extent of aortic involvement.

In this study claustrophobia prevented a few patients from having a magnetic resonance imaging scan. Of those who had both scans performed $5 / 12$ patients preferred the echocardiogram, but were able to tolerate the magnetic resonance imaging scanner. Both scans took approximately 30 minutes to perform. Magnetic resonance imaging scanning is contraindicated in patients with pacemakers and metal prostheses; echocardiography is possible in such cases.

\section{Conclusions}

We conclude that measuring aortic distensibility and stiffness index provides additional information on the involvement of the aorta in patients with Marfan's syndrome. The usefulness of these measurements in predicting risk of aortic dissection or rupture is yet to be established. Magnetic resonance imaging gives good quality images of the whole thoracic aorta, measurements are reproducible and most patients tolerate it. If magnetic resonance imaging is unavailable echocardiography provides an adequate alternative and may be more suitable for some patients. If serial measurements are to be made one technique should be chosen and used on each occasion. 
1 Roberts WC, Honig HS. The spectrum of cardiovascular disease in the Marfan's syndrome: A clinico-morphologic study of 18 necropsy patients and comparison to 151 previously reported necropsy patients. Am Heart $\mathcal{F}$ 1982;104:115-135.

2 Bruno L, Tredici S, Mangiavacchi M, Colombo V, Mazzotta GF, Sirtori CR. Cardiac, skeletal and ocular abnormalities in patients with Marfan's syndrome and their relatives. Comparison with the cardiac abnormalitheir relatives. Comparison with the cardiac abnormali-
ties in patients with kyphoscoliosis. Br Heart $\mathcal{f} 1984$; ties in patient $220-30$.
51:22

3 Hirata K, Triposkiadis F, Sparks E, Boudoulas H, Wooley C. The Marfan syndrome: Cardiovascular physical findings and diagnostic correlates. Am Heart $\mathcal{f}$ 1992;123: ings and

4 Hollister DW, Goodfrey M, Sakai LY, Pyeritz RE. Immunohistologic abnormalities of the microfibrillarfibre system in the Marfan's syndrome. $N$ Engl f Med 1990;323:152-9.

5 Murdoch JL, Walker B, Halpern BL, Kuzma JW, McKusick VA. Life expectancy and causes of death in the Marfan's syndrome. N Engl $\mathcal{F}$ Med 1972;286:804-8.

6 Detrano RD, Moodie DS, Gill CC, Markovich D, Simpfendorfer C. Intravenous digital subtraction aortography in the preoperative and postoperative evaluation of Marfan's aortic disease. Chest 1985;88:249-53.

7 Schaefer S, Peshock RM, Malloy CR, Katz J, Parkley JT, Wilnerson JT. Nuclear magnetic resonance imaging in Marfan's syndrome. $₹ \mathrm{Am}$ Coll Cardiol 1987;9:70-4.

8 Stefanadis C, Stratos C, Boudoulas H, Kouroukli C, Toutouzas P. Distensibility of the ascending aorta: comparison of invasive and non-invasive techniques in parison of invasive and non-invasive techniques in healthy men and in men with

9 Kawasaki T, Sassayama S, Yagi S, Asakawa T. Noninvasive assessment of the age related changes in stiffness of major branches of the human arteries. Cardiovasc Res 1987;21:678-87.
10 Hirata K, Triposkiadis F, Sparks E, Bowen J, Wooley CF, Boudoulas H. The marfan's syndrome: abnormal aortic elastic properties. $₹ \mathrm{Am}$ Coll Cardiol 1991;18:57-63.

11 Salvolainen A, Keto P, Hekali P, Nisula L, Kaitila I, Vitasalo $M$, et al. Aortic distensibility in children with the marfan's syndrome. Am ₹ Cardiol 1992;70:691-3.

12 Beighton P, de Paepe A, Finidori DG. International nosology of heritable diseases of connective tissue. Berlin 1986. Am $\mathcal{F}$ Med Genet 1988;29:581-94.

13 Mohiaddin RH, Underwood SR, Bogren HG, Firmin DN, Kilpstein RH, Rees RSO, Longmore DB. Regional aortic Kilpstein RH, Rees RSO, Longmore DB. Regional aortic compliance studied by magnetic resonance imaging: the effects of age, training and coronary artery disease. $\mathrm{Br}$
Heart $\mathcal{} 1989 ; 62: 90-6$. Heart $f 1989 ; 62: 90-6$.

14 Francke U, Furthmayr H. Marfan's syndrome and other disorders of fibrillin. N Engl f Med 1994;19:1384-5.

15 Shores J, Berger K, Murphy EA, Pyeritz RE. Progression of aortic dilatation and the benefit of long-term $\beta$-adrenergic blockade in Marfan's syndrome. $N$ Engl f Med 1994;19:1335-41.

16 Crawford ES. Marfan's syndrome; broad spectral surgical treatment of cardiovascular manifestations. Ann Surg 1983;198:487-505.

17 Gott VL, Pyeritz RE, Cameron DE, Greene PS, McKusick VA. Composite graft repair of Marfan aneurysm of the ascending aorta: results in 100 patients. Ann Thorac Surg 1991;52:38-45.

18 Murgatroyd F, Child A, Poloiecki J, Treasure $T$, Pumphrey CW. Does routine echocardiographic assessment of the aortic root diameter help predict the risk of ment of the aortic root diameter help predict the risk of
dissection in the Marfan syndrome? [Abstr.] Eur Heart $f$ 1991;12(suppl):410.

19 Treasure $T$. Elective replacement of the aortic root in Marfan's syndrome. Br Heart f 1993;69:101-3.

20 Schlatmann TJ, Becker AE. Pathogenesis of dissecting aneurysm of aorta. Am $\mathcal{F}$ Cardiol 1977;39:21-6.

21 Roberts WC. Aortic dissection: anatomy consequence and cause. Am Heart f 1981;101:195-214. 